\title{
On the suppression of the sidelobes of the supercurrent in small Josephson tunnel junctions
}

\author{
E.P. Houwman, J.G. Gijsbertsen, J. Flokstra and H. Rogalla \\ University of Twente, Faculty of Applied Physics, PO Box 217.7500 AE Enschede, Netherlands
}

Received 4 September 1991

Revised manuscript received 5 October 1991

\begin{abstract}
The critical currents of $\mathrm{Nb} / \mathrm{Al}, \mathrm{Al}$-oxide, $\mathrm{Al} / \mathrm{Nb}$ tunnel junctions of various shapes have been measured as a function of the applied magnetic field. For the square junction and for some special shapes like the diamond, " $1+$ cosine" and quartic junctions the $I_{c}(B)$ pattern falls off theoretically as $1 / B^{n}$, with $n$ respectively equal to $1,2,3$ and 4 . In general the measurements are in good agreement with the theoretical predictions. For the " $l+$ cosine" and quartic shapes we found a sidelobe suppression that is even larger than that obtained in theory. For the quartic junction the first sidelobe is only $0.3 \%$ of the zero-field current. An $I_{c}(B)$ modulation with a small, only slowly decreasing amplitude is observed for the diamond, " $1+$ cosine", and quartic junctions, that can be explained by rounding of the sharp edges of the junction shapes, due to the fabrication process.
\end{abstract}

\section{Introduction}

In many applications of Josephson tunnel junctions there is a need to suppress the sidelobes of the Josephson current. We develop tunnel junctions for the detection of X-rays for applications is astronomy. These devices are often current biased in the subgap, and for stable operation it is necessary that the rate of switching to the zero-voltage state is small. This can be achieved by suppressing the supercurrent below the biasing current by means of a magnetic field. For a rectangular junction this results in the well-known Fraunhofer pattern, that falls off as $1 / B$ with the magnetic field.

It is already known for a long time that the shape of small Josephson junctions can be designed so as to manipulate the critical current pattern $I_{\mathrm{c}}(B)$ [1]. Recently, Peterson has worked out this idea in order to obtain large suppression of the sidelobes [2]. He calculated for some rather simple shapes of the junction a sidelobe pattern that varies as $1 / B^{n}$, with $n$ up to 4 . This means a theoretical suppression of the first sidelobe below $1 \%$ of the zero-field current.

In this paper we present our first experimental results on the suppression of the supercurrent in small Josephson junctions of various shapes, for which the
$I_{\mathrm{c}}(B)$ pattern falls off as $1 / B, 1 / B^{2}, 1 / B^{3}$, and $1 / B^{4}$.

\section{Theory}

It is well-known that if the length $L$ of a Josephson junction normal to an applied magnetic field is less than about twice the Josephson penetration length, $\lambda_{\mathrm{J}}=\left(\Phi_{0} / 2 \pi \mu_{0} d J_{\mathrm{c}}\right)^{1 / 2}$, the self-induced magnetic field has a negligible effect on the critical current distribution in the junction and thus on the $I_{\mathrm{c}}(B)$ pattern. Here $\Phi_{0}$ is the flux quantum, $d$ is equal to $2 \lambda_{\mathrm{L}}+t$, with $\lambda_{\mathrm{L}}$ the London penetration depth into the superconductor and $t$ the thickness of the oxide barrier, and $J_{\mathrm{c}}$ the maximum critical current density. It was shown that for such "small" junctions, which are symmetrically shaped, the total current through the junction is given by [2]

$I_{\mathrm{c}}(B)=2 J_{\mathrm{c}} \sin \left(\phi_{0}\right) \int_{-x_{0}}^{x_{0}} f(x) \cos (k x) \mathrm{d} x$.

Here it is assumed that the midpoint of the junction coincides with the centre of the coordinates (see fig. 1); $\phi_{0}$ is a phase constant chosen to maximize the critical current. The even function $y=f(x)$ describes 


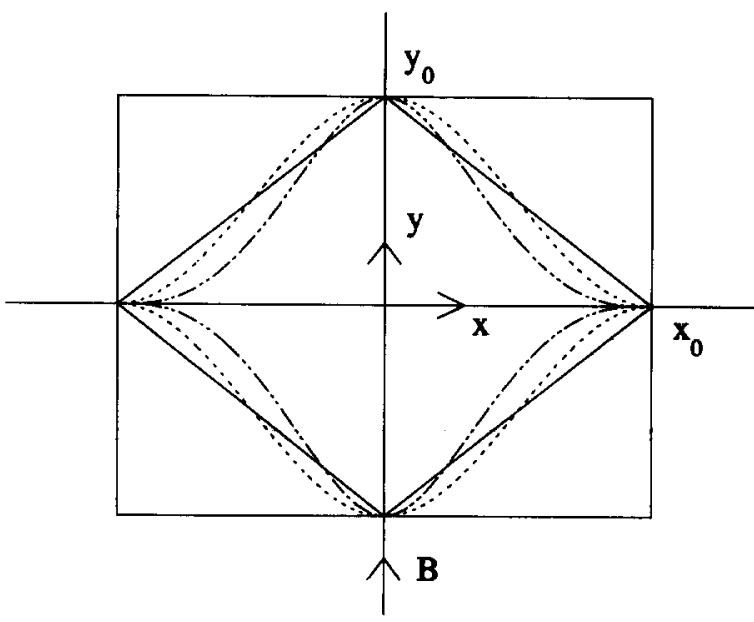

Fig. 1. Shapes of the rectangular, diamond $(-), " 1+\operatorname{cosine"~}(\cdots)$ and quartic (-..-) junctions.

the boundary of the junction for $y>0$, and $-f(x)$ describes the bottom half; $2 x_{0}=L$ and $2 y_{0}=2 f(0)$ are respectively the maximum dimensions in the $x$ and $y$-direction. The wavevector is given by $k=2 \pi B d / \Phi_{0}$, where $B$ is the constant magnetic field applied in the $y$-direction (see fig. 1). Thus the total current is proportional to the cosine Fourier transform of the junction shape.

Here we consider the following junction shapes.

(1) The rectangular junction with $f(x)=y_{0}$, gives

$I_{\mathrm{c}}(B)=I_{m}\left|\frac{\sin (\pi b)}{\pi b}\right| \sim 1 / B$,

where $b=B / B_{0}, B_{0}=\Phi_{0} / L d$ and $I_{\mathrm{m}}=J_{\mathrm{c}} A . A$ is the junction area.

(2) The diamond, $f(x)=y_{0}\left(1-|x| / x_{0}\right)$, gives

$I_{\mathrm{c}}(B)=I_{\mathrm{m}}\left|2 \frac{1-\cos (\pi b)}{(\pi b)^{2}}\right| \sim 1 / B^{2}$.

(3) The " $1+\operatorname{cosine",~} f(x)=\left(y_{0} / 2\right)(1+\cos (\pi x)$ $\left.x_{0}\right)$ ), gives

$I_{\mathrm{c}}(B)=I_{\mathrm{m}}\left|\frac{\sin (\pi b)}{\pi b\left(1-b^{2}\right)}\right| \sim 1 / B^{3}$.

(4) The quartic polynomial, $f(x)=y_{0}(1-$ $\left.6\left(x / x_{0}\right)^{2}+8\left(|x| / x_{0}\right)^{3}-3\left(x / x_{0}\right)^{4}\right)$, gives

$$
\begin{aligned}
& I_{\mathrm{c}}(B)=I_{\mathrm{m}}\left|30 \frac{-6 \sin (\pi b)+2 \pi b \cos (\pi b)+4 \pi b}{(\pi b)}\right| \\
& \sim 1 / B^{4} .
\end{aligned}
$$

The last two shapes have zero curvature at $x= \pm x_{0}$, thus giving pointed ends. We use standard photo lithography and anodization for defining the shape of the junction. This process has a resolution of about $0.5-1 \mu \mathrm{m}$; therefore the sharp edges of the junctions are rounded off. In a first approximation this can be modelled by placing the shapes on a pedestal, such that $f\left( \pm x_{0}\right)=p y_{0}$. The functions describing these shapes may have the form $g(x)=(1-p) f(x)+p y_{0}$. It is straightforward to show that this leads to the critical current dependence

$$
\begin{aligned}
& I_{\mathrm{c}, \mathrm{ped}}(B) \\
& \quad=I_{\mathrm{m}}\left|\frac{A_{1}}{A} i_{\mathrm{c}}(b)+\frac{A_{2}}{A} \frac{\sin (\pi b)}{\pi b}\right|,
\end{aligned}
$$

with $A_{1} / A=(1-p) /(1+p)$ and $A_{2} / A=2 p /(1+p)$ for the diamond and " $1+$ cosine" junctions and $A_{1} /$ $A=(0.4-0.4 p) /(0.4+0.6 p)$ and $A_{2} / A=p /$ $(0.4+0.6 p)$ for the quartic junction. Here $i_{\mathrm{c}}(b)$ is given by the function between the modulus bars in the eqs. (2)-(5). In case the first term of the right hand side of eq. (6) falls off rapidly with increasing $B$, the second term, which falls off only slowly, will dominate for large $B$. As was already pointed out by Peterson, the pedestal can be chosen such that the close-in lobes are suppressed additionally. However, it should be mentioned that the close-in lobes of junction shapes with high sidelobe suppression are very sensitive for the detailed shape of the junction at $x$ near $\pm x_{0}$. The overall shapes of the main $I_{c}(B)$ lobes are not very sensitive to the shapes of the various junctions, apart from the tails at low $I_{\mathrm{c}}(B)$ values and the field for which the first $I_{\mathrm{c}}(B)$ minima are reached.

\section{Fabrication}

We prepared Josephson junctions in $\mathrm{Nb} / \mathrm{Al}, \mathrm{Al}-$ oxide, $\mathrm{Al} / \mathrm{Nb}$ technology [3], with the shapes mentioned above and of various dimensions. Here we will only consider junctions (with largest dimensions of 
about $55 \mu \mathrm{m}$ ) for which the curved shapes could be made fairly accurately.

A multilayer is deposited and structured by liftoff as a square with sides of $70 \mu \mathrm{m}$. The junction shapes are defined by the SNAP process and have maximum dimensions of about $55 \mu \mathrm{m}$ in the $x$ - and $y$-directions. The counter electrodes is $60 \mu \mathrm{m}$ square. In order to avoid distortion of the applied field as much as possible the $5 \mu \mathrm{m}$ wide current leads are attached to the middles of the two sides, which are parallel to the field direction.

The lithographic contact printing mask for the definition of the junction areas was made in two steps. The first mask was made with a tenfold expansion of the junction shapes, described by the functions $f(x)$ given above, that were built up of rectangles with a minimum size of $5 \mu \mathrm{m}$ and with arbitrary orientation. This mask was copied on the final contact mask with a tenfold reduction.

The critical current density is about $135 \mathrm{~A} / \mathrm{cm}^{2}$, giving a $\lambda_{\mathrm{J}}$ of about $35 \mu \mathrm{m}$. Thus $2 \lambda_{\mathrm{J}}$ is larger than the junction dimensions perpendicular to the magnetic field.

\section{Results}

The junctions are mounted on a holder that is placed on the axis of a solenoid. This assembly is placed inside a superconducting can, that is inside a $\mu$-metal can. The junctions are cooled down in zero magnetic field. All measurements were done at about $1.6 \mathrm{~K}$.

The experimental $I_{\mathrm{c}}(B)$ pattern of a square junction is given in fig. 2 , together with a fit according to eq. [2]. The theoretical curve fits the data reasonably well (also for reduced critical currents larger than 0.5 ). This indicates that the conditions for eq. (2) are fulfilled, i.e. a nearly constant critical current density of the junction and a non-distorted, constant magnetic field, that is perpendicular to the junction side. We can explain the deviations from the fit by a small defect with higher conductivity in the junction. From the minima of the pattern the scaling field current $I_{0}$, that is linear proportional to $B_{0}=\Phi_{0} / L d$, is obtained. This value is used in the fits for the $I_{c}(B)$ patterns of the other junctions. In the next figures we show only the low current parts of the $I_{\mathrm{c}}(B)$ patterns, as these are the most sensitive to the shapes of the various junctions. It is found that the main lobes are very well described by the various models.

Figure 3 shows the $I_{\mathrm{c}}(B)$ pattern of the diamond type junction with equal sides that are orthogonal to each other. The theoretical curve (eq. (3)) is obtained by taking $I_{\mathrm{m}}$ equal to the maximum critical current at zero field and the current period equal to $I_{0}$ (square) $\times L$ (square) $/ L$ (diamond). The first three sidelobe maxima are fairly well described by the fit from eq. (3), with maxima that are respectively $5.0 \%$, $1.6 \%$, and $0.7 \%$ of the zero field current. However, between these large lobes small lobes show up and beyond the third sidelobe the suppression is much larger than predicted. Further, at high magnetic fields the $I_{c}(B)$ pattern shows a modulation with a small, nearly constant amplitude and a period of about 0.36 A which is half the period of the large amplitude modulation (see the inset of fig. 3 ). A period of 0.36 A corresponds to a dimension perpendicular to the field of about $57 \mu \mathrm{m}$. This value is nearly equal to the designed length $2 x_{0}=56 \mu \mathrm{m}$ of the diamond. These effects can be explained by the introduction of a pedestal (which can easily be caused by the rounding), as described by eq. (6). From the amplitude of the short period modulation we deduced a value $p \approx 0.01$, which corresponds to a total pedestal height of about $0.6 \mu \mathrm{m}$. This value is of the order of the rounding error due to the lithographic and anodization processes. The fit in the inset, obtained from the second term in eq. (6), using this $p$ value, is fairly good.

Figure 4 shows the experimental $I_{c}(B)$ curve for the " $1+$ cosine" junction, as well as the theoretical curve obtained from eq. (4). All sidelobe amplitudes are below $1 \%$ of the main lobe. This is even more than predicted by theory. The reduction of the amplitudes cannot be described by a simple pedestal. For higher fields we observe again a modulation with a small, but slightly varying amplitude and period, indicating the presence of a pedestal. From this modulation we calculated tentatively a $p$ value of about 0.007 and an average period of $0.38 \mathrm{~A}$, corresponding to a length of $54 \mu \mathrm{m}$. This value is equal to the designed maximum $x$-dimension.

Figure 5 shows the experimental $I_{\mathrm{c}}(B)$ curve of the quartic junction, together with the theoretical 


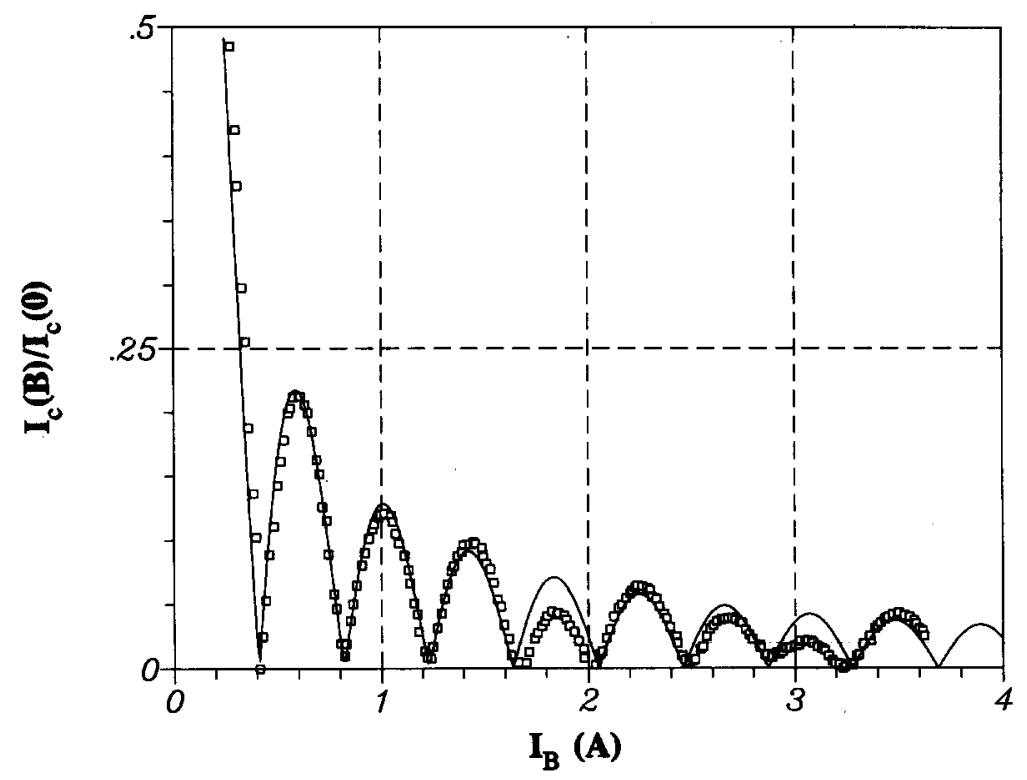

Fig. 2. Reduced critical current $I_{\mathrm{c}}(B) / I_{\mathrm{c}}(0)$ of the rectangular junction. (Experimental data ( $\square$ ); theoretical fit from eq. (2) (-).)

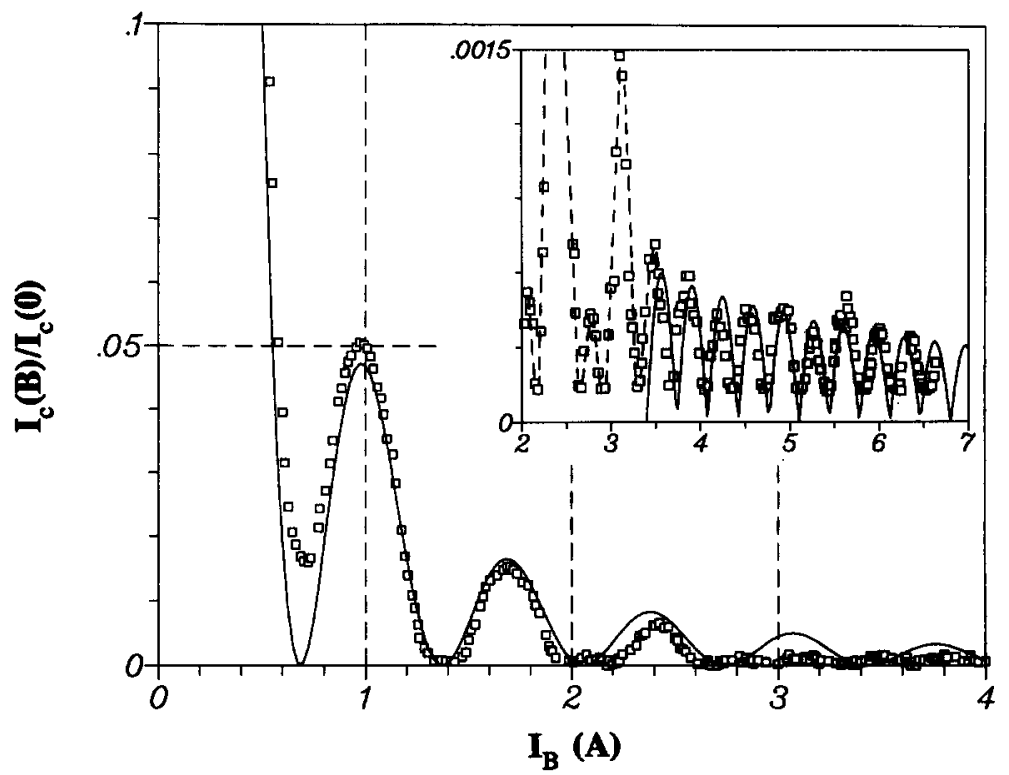

Fig. 3. $I_{c}(B)$ pattern of the diamond shaped junction. (Experimental data $(\square)$; theoretical fit from eq. (3) (-).) The inset shows an expansion of the $I_{c}(B)$ pattern at higher magnetic fields. (Experimental data $(\square)$; fit with the $|\sin (\pi b) /(\pi b)|$ term of eq. (6) $(-)$; the dashed line is a guide for the eye.)

curve according to eq. (5). The experimental curve shows a shoulder, that is much smaller than the one predicted by the theoretical curve. Two sidelobes with an amplitude of only $0.25 \%$ appear, which is much smaller than the theoretical amplitude of the first sidelobe. At higher fields a slowly falling modulation 


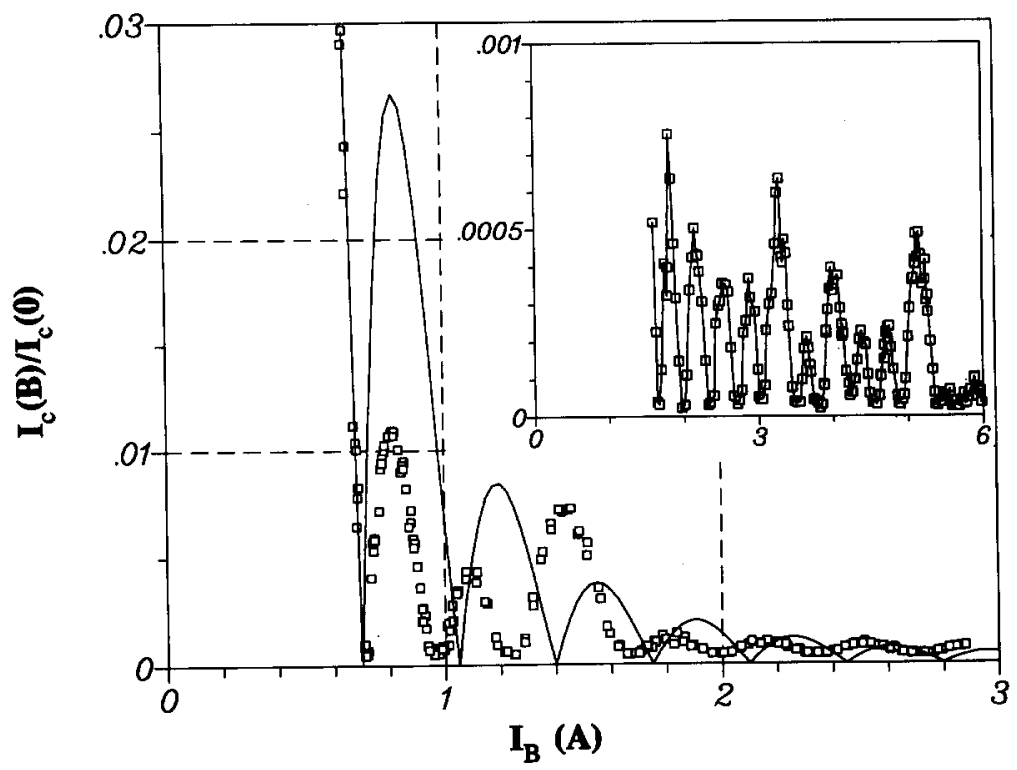

Fig. 4. $I_{\mathrm{c}}(B)$ pattern of the " $1+$ cosine" junction. (Experimental data $(\square)$; theoretical fit from eq. (4) (-)) In the inset an expansion of the high field part of the $I_{c}(B)$ pattern. The full curve line is a guide for the eye.

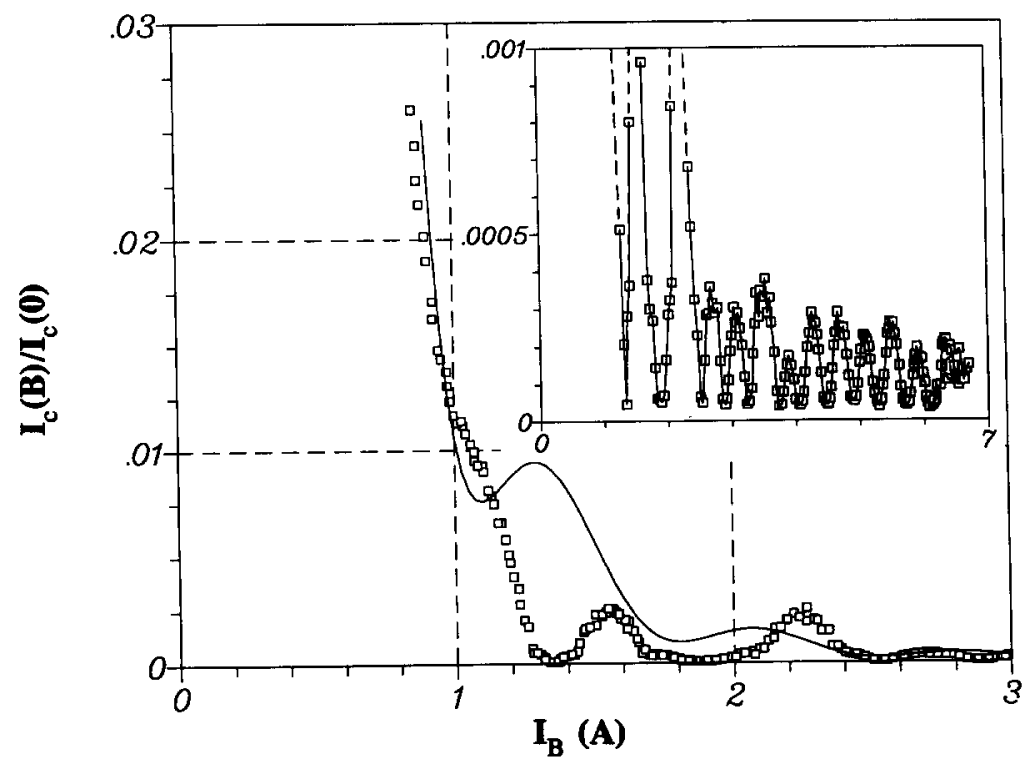

Fig. 5. $I_{c}(B)$ pattern of a quartic junction. (Experimental data $(\square)$; theoretical fit from eq. [5] (-).) The full curve in the inset is a guide for the eye.

is present with a period of $0.39 \mathrm{~A}$, indicating the presence of a small pedestal with $p=0.005$. Again we ascribe the large deviations of the experimental $I_{\mathrm{c}}(B)$ curve from theory to the rounding of the junction shape. 


\section{Conclusions}

We showed experimentally that it is possible to obtain suppression of the sidelobes of the $I_{\mathrm{c}}(B)$ pattern below $1 \%$ of the zero-field supercurrent for rather simple junction shapes, like the " $1+$ cosine" and the quartic junctions.

For applications where large area junctions are needed, this is a very good method to suppress the sidelobes, without the need of a large magnetic field or accurate tuning of the field strength in a minimum of the Fraunhofer pattern. This property is especially interesting for applications were a large field has a deteriorating effect on the device performance, as is probably the case in X-ray detectors [4], or where arrays of junctions are needed [5]. In the latter case it is not necessary to reproduce the dimensions of the junctions very accurately, in order to be able to suppress the supercurrent.

To describe accurately the experimental $I_{c}(B)$ patterns of the various junctions one needs a detailed description of the actual junction shapes, that is of the function $f(x)$. However, it appears that some rounding errors do not increase the sidelobe ampli- tudes, but on the other hand suppress the close-in sidelobes even more. In a first approximation the effect of the rounding can be described by the introduction of a small pedestal. This gives a fairly good explanation for the high field behaviour of the $I_{\mathrm{c}}(B)$ pattern.

For the operation of a junction as an X-ray detector not only the $I_{\mathrm{c}}(B)$ patterns are of importance, but also the Fiske resonances, that are present in these devices. The results of that study will be presented in a separate paper.

\section{References}

[1] T. Van Duzer and C.W. Turner, Principles of Superconductive Devices and Circuits (Elsevier, New York, 1981) section 4.05.

[2] R.L. Peterson, Cryogenics 31 (1991) 132.

[3] J. Flokstra, D.J. Adelerhof, E.P. Houwman, D. Veldhuis and H. Rogalla, Clin. Phys. and Phys. Meas. (1991) to be published.

[4] K.S. Wood, M.N. Lovelette and D. van Vechten, IEEE Trans. Magn. MAG-27 (1991) 2673.

[5] M. Kurakudo, A. Matsumura and T. Takahashi, Rev, Sci. Instrum. 62 (1991) 156. 